\title{
A HYBRID METHOD TO COMBINE WAVE PENETRATION AND LOCAL WAVE GROWTH IN LARGE HARBOR BASINS
}

\author{
Gerbrant Ph. van Vledder',
}

\begin{abstract}
This paper describes a hybrid method to determine the wave conditions in large harbor basins were wave propagation and local wave growth by wind are both of relevance. The method was developed to include the effects of diffraction and wind wave growth in the assessment of design wave conditions that so far cannot be computed within one wave model. The diffraction effects are computed with a phase-resolving wave penetration model, and the wind effect is computed with a phase averaged spectral wave model. The method accounts for the effect the local wind wave growth occurs on top of the penetrating wave field. To isolate the wind wave growth, two model runs are performed with the phase-average model. One run includes wind forcing, whereas in the other run wind forcing is switched off. Subtracting the results of both methods isolates the local wave growth. The method is illustrated with a hypothetical example for the Port of IJmuiden, located along the North Sea coast of the Netherlands. In addition, an overview is given of the physical processes relevant for the determination of design wave conditions in large harbor basins. The assumptions of the hybrid method are discussed and recommendations are given for further improvements.
\end{abstract}

Keywords: numerical wave modelling; SWAN; PHAROS; mild slope model, wave penetration; diffraction, wave growth

\section{INTRODUCTION}

For the design of water defenses and structures in harbor basins, offshore wave conditions need to be transformed to wave conditions in the harbor basin. The offshore wave conditions are usually based on a statistical analysis of measured or predicted wave conditions and expressed in terms of a significant wave height, a mean wave period and wave direction. Commonly used tools to assess the amount of wave penetration are physical model experiments and numerical phase-resolving models (Demirbilek et al., 2010). These models are able to accurately determine the amount of wave penetration while accounting for the effects of transmission, reflection, diffraction, shoaling and dissipation.

These methods, however, lack the effect of local wind wave growth which can be of significant influence in large harbor basins. As waves penetrate into a harbor basin they remain under the influence of the local wind, maintaining an influx of wave energy to the existing wave field. Neglecting this effect may lead to serious errors in large harbor basins, all the more as design conditions are usually associated with 'extreme' wind speeds. It is therefore needed to include the effect of local wave growth into the methodology by combining these effects with those of wave penetration.

Presently, no single wave model exists that is able to combine, among other processes, the effects of diffraction and wind wave growth. Phase-resolving models are able to determine the effects of wave propagation, reflection, refraction, transmission, diffraction, shoaling and dissipation effects, but not the effect of wind wave growth. Conversely, spectral phase-averaged methods are able to include wind wave growth, and all the previously mentioned effect except for diffraction. For many applications, a need exists to include all of these effects in the wave modelling approach. Therefore, a hybrid method was developed in the Netherlands to combine the effect of wave penetration and local wave growth using a mix of wave models results.

When offshore waves penetrate into a harbor basin, they encounter a plethora of effects. Close to shore they often propagate through or over a dredged entrance channel where channel refraction and bottom friction dissipation in shallow areas play a role. Near the harbor entrance breakwaters reflect, dissipate and transmit wave energy thereby providing a certain amount of protection. The effectiveness of the breakwater depends on the wave characteristics as well as the relative freeboard, defined as the ratio of the difference of height of the structure minus the water level, divided by the incident significant wave height. Inside the harbor basin, the penetrated waves may encounter dams, quays, depth variations and flooded harbor areas leading to refraction along the entrance channel, reflection against dams and quays, and shoaling and dissipation in shallow areas. In addition, at higher water levels transmission over dams and at the edges of flooded areas also needs to be accounted for.

As said before, in case of design conditions, the associated wind may lead to additional wave growth inside the harbor basin. Such effects can be included in different ways. A simple approach is to apply parametric wind wave growth relations to compute the effect of local wave growth and to add this result to the results of the wave penetration model. A complication of such a method is the determination of the wind direction dependent effective fetch lengths. Moreover, the starting point of such a fetch may be in the opening between the breakwaters or an upwind land/harbor boundary. Further, the starting point of such a fetch computation cannot always be taken as a zero condition as already waves exist in the harbor opening or behind the breakwater were wave transmission effects

\footnotetext{
${ }^{1}$ Van Vledder Consulting, Van Oldenbarneveltstraat 76, 8121 ZJ Olst, The Netherlands. gerbrant@ vanvledderconsulting.com

${ }^{2}$ Civil Engineering and Geosciences, Delft University of Technology, P.O. Box 5048, 2600 GA Delft, The Netherlands,
} 
occur. For small harbor basins, it is expected that wind effects are of minor importance, whereas for large harbor basins, wind effects will become increasingly important the further the (downwind) location in a harbor basin, especially for design conditions.

The specification of the effective wind in a harbor basin is a problem in itself. As harbor basins are often situated inland, i.e. behind the coastline, the atmospheric flow may be dampened by the surrounding land where surface friction effects reduce the effectively wind speed. Neglecting these friction effects may then yield a small overestimation (order of 10\%) in wind speed. This reduction is direction dependent and usually the smallest when the wind blows along the main axis of the harbor basin. Omitting this possible reduction should be considered in the design process.

A common method to assess the required strength or height of water defenses in the Netherlands is based on a probabilistic approach by considering the contribution of waves generated for a range of wind speeds, wind directions and water levels. A key part of this approach is to determine the wave conditions for a matrix of conditions, with dimensions for wind speed, wind direction and water level. A typical approach is to consider 12 wind directions, 8 wind speeds and 6 water levels, resulting in a set of at most 576 conditions. As some combinations are unlikely to occur, e.g. a wind blowing from land to sea in combination with a high water level, the final number will be reduced somewhat. For each of these combinations the associated offshore wave conditions need to be specified, e.g. by a wave climate analysis.

An interesting feature of this approach is that a range of water levels is considered. As a consequence various low-lying harbor areas may be flooded, effectively changing the extent of the area to be modelled. Flooding and drying should then be accounted for in the applied wave models.

From a numerical modelling of view it is important to choose the right computational model or set of models to transform the offshore wave conditions to output location in front of the water defenses and structures. These models should at least be able to process the relevant wave related physical processes; refraction, reflection, transmission, diffraction, shoaling, dissipation and local wave growth. Unfortunately, no single wave model yet exists that can model all these relevant effects simultaneously, of which the simultaneous effects of diffraction and local wave growth are the most problematic.

In open water wind wave growth can accurately be determined using phase-averaged wave models, but they are less suited in partly sheltered areas like harbor basins as they are not (yet) able to account for local effects of diffraction. Despite attempts to include diffraction in SWAN (Holthuijsen et al., 2003) it is not recommended to use this option as the approximation is rather crude. In contrast, phase-resolving models are (still) not able to account for effects of wind although some attempts have been made (e.g. Chen et al., 2004). So, the key problem in choosing the proper numerical modelling tools, is that no single model exists that, among other processes, can account for both the effects of diffraction and wind wave growth.

To solve this shortcoming, a hybrid modelling technique was developed in the Netherlands to combine the results of both types of models to assess the combined effect of wave diffraction and local wave growth, as well as refraction, reflection, transmission, shoaling and dissipation to determine wave conditions in large harbor basins. As the factual origin of the hybrid method was never properly documented, this paper summarizes the basic concepts of the hybrid method.

The hybrid method is illustrated for the Port of IJmuiden located along the North Sea coast with the SWAN wave model (Booij et al., 1999, SWAN team, 2014) the mild slope model PHAROS (Hurdle et al., 1989, and the online documentation https://www.deltares.nl/en/software/pharos). The bathymetry of the Port of IJmuiden and part of the offshore area is shown in Figure 1. This figure shows the offshore entrance channel continuing towards the eastern end of the harbor basin. The eastern edge of the harbor basin consists of a set of ship locks, connecting the North Sea to the North Sea Channel towards Amsterdam. The harbor entrance is partly sheltered with two large breakwaters. The east-west distance is about $5 \mathrm{~km}$, such that effects of local wave growth are expected to become relevant under strong wind conditions, especially as dominant wind and wave directions are from westerly directions.

The setup of this paper is as follows. First, the basic concepts of the hybrid method are introduced, followed by results of a hypothetical application for the Port of IJmuiden. Hereafter, the inherent assumptions of the hybrid method are discussed. Finally some conclusions and recommendations are provided. 


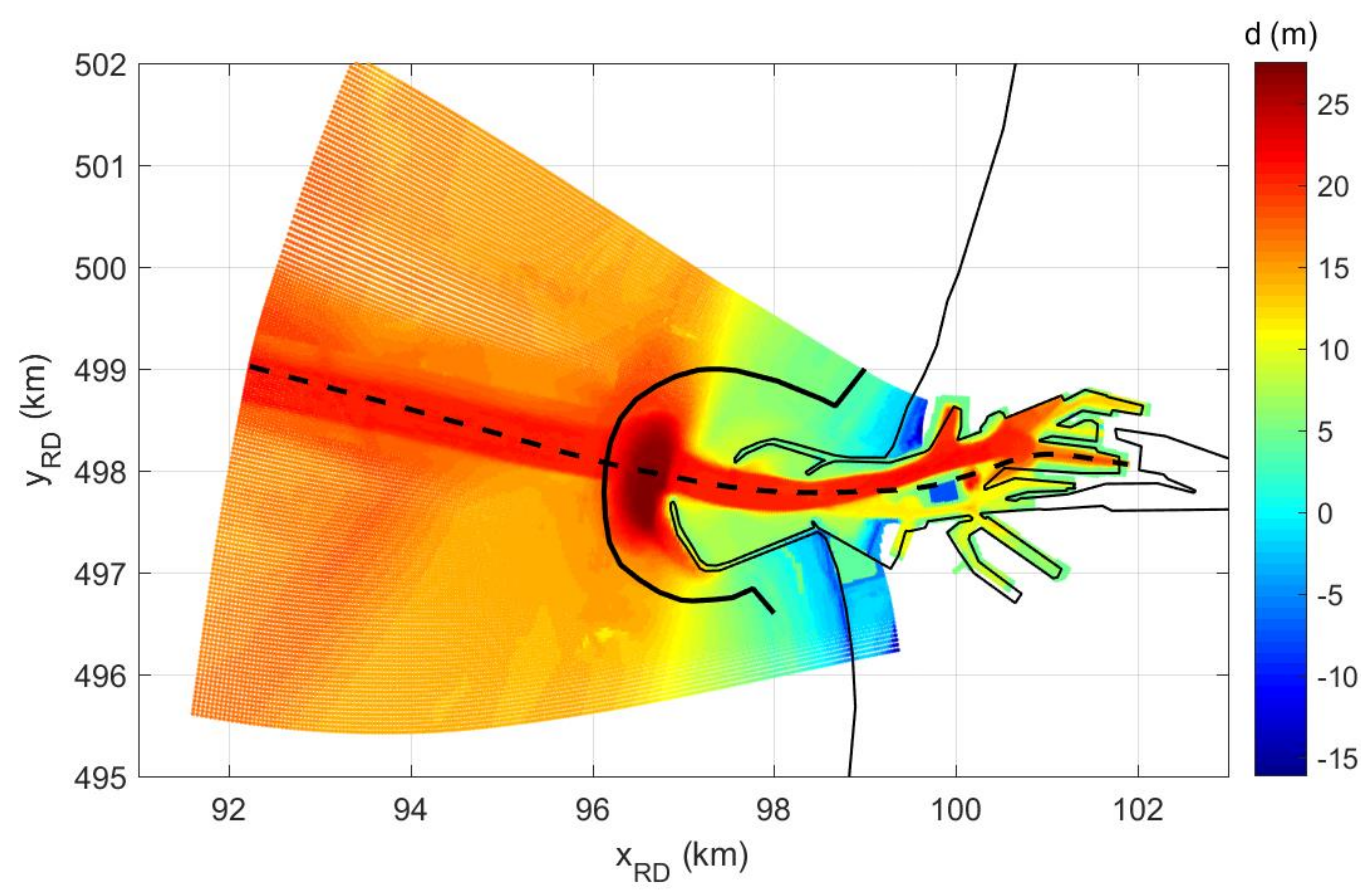

Figure 1 - Bathymetry of the Port of IJmuiden, as displayed on the SWAN computational grid. The dashed line indicates the channel axis and the rounded black line indicates the boundary of the PHAROS wave penetration model.

\section{METHOD DESCRIPTION}

The basic idea of the hybrid method is to add the effect of local wind wave growth to the penetrated wave field. This is achieved by performing three different wave model runs; one run with a phase-resolving wave model to determine the amount of wave penetration including diffraction effects and two runs with a phase-averaged wave model to determine the contribution of the wind on top of the penetrating waves. The wave boundary conditions for each of the model runs are equal to ensure consistency. The result of wave penetration can generally be expressed in terms of spectral moments of the wave variance $E$ :

$$
E_{P}(x, y)
$$

where the subscript $\mathrm{P}$ refers to wave Penetration and where $\mathrm{x}$ and $\mathrm{y}$ refer to the spatial coordinates. The effect of wind is obtained by performing two runs with a phase-averaged wind wave model, both with the same wave boundary condition as for the wave penetration run. The first wind model run includes wind (W), whereas in the second run wind forcing is deactivated ( $\mathrm{N}$ of No wind). The result of the wind model run with wind activated can be expressed as:

$$
E_{W}(x, y)
$$

where the subscript $\mathrm{W}$ indicates the effect of Wind. The results of the wind wave model run with wind deactivated $(\mathrm{N})$ can be expressed as:

$$
E_{N}(x, y)
$$

where the subscript $\mathrm{N}$ indicates No wind. The contribution of additional wind generation $\mathrm{G}$ can then simply be expressed as the difference between the results of the model runs with wind $(\mathrm{W})$ and without wind $(\mathrm{N})$ :

$$
E_{G}(x, y)=\mathrm{E}_{W}(x, y)-E_{N}(x, y)
$$


where the subscript $G$ indicates the additional Growth of waves. In the last step, the wind contribution $G$ is added to the results of the phase-resolving model. Thus the total wave condition can be expressed as:

$$
\begin{aligned}
E_{T} & =E_{P}+E_{G} \\
& =E_{P}+\left(E_{W}-E_{N}\right)
\end{aligned}
$$

The expressions (1) to (5) are given in the general notation of variances E (equivalent to wave energy, apart from a factor $\rho_{\mathrm{w}} \mathrm{g}$ ). To compute the associated significant wave heights and spectral periods, the expressions can also be replaced by expressions in term of spectral moments. The equations in terms of wave energy or spectral moments can also be expressed in terms of the significant wave height $\mathrm{H}_{\mathrm{m} 0}$ and the mean spectral period $\mathrm{T}_{\mathrm{m}-10}$. To that end, use is made of the spectral moments $m_{0}$ and $m_{-1}$ of the wave spectrum $E(\mathrm{f})$ :

$$
m_{n}=\int f^{n} E(f) d f
$$

The significant wave height $H_{\mathrm{m} 0}$ and the spectral wave period $T_{\mathrm{m}-1,0}$ are defined as:

$$
\begin{aligned}
H_{m 0} & =4 \sqrt{m_{0}} \\
T_{m-1,0} & =\frac{m_{-1}}{m_{0}}
\end{aligned}
$$

Inversely, the spectral moments $m_{0}$ and $m_{-1}$ can also be expressed in terms of the significant wave height $H_{\mathrm{m} 0}$ and the spectral wave period $T_{\mathrm{m}-1,0}$ :

$$
\begin{aligned}
& m_{0}=\frac{1}{16} H_{m 0}^{2} \\
& m_{-1}=\frac{1}{16} H_{m 0}^{2} T_{m-1,0}
\end{aligned}
$$

The amount of wave growth $G$ can then be expressed in terms of the significant wave height $H_{\mathrm{m} 0}$ and the spectral wave period $T_{\mathrm{m}-1,0}$ (the sub- and superscripts refer to the type of wave model computation):

$$
\begin{aligned}
H_{m 0, G} & =4 \sqrt{m_{0}^{W}-m_{0}^{N}} \\
& =\sqrt{H_{m 0, \mathrm{~W}}^{2}-H_{m 0, \mathrm{~N}}^{2}} \\
T_{m-1,0, G} & =\frac{\left(m_{-1}^{W}-m_{-1}^{N}\right)}{\left(m_{0}^{W}-m_{0}^{N}\right)} \\
& =\frac{\left(H_{m 0, W}^{2} T_{m-10, W}-H_{m 0, \mathrm{~N}}^{2} T_{m-10, \mathrm{~N}}\right)}{\left(H_{m 0, \mathrm{~W}}^{2}-H_{m 0, \mathrm{~N}}^{2}\right)}
\end{aligned}
$$

Combination of the expressions (5) and (9) yields the following expressions to determine the combined effect (or total: T) significant wave height $H_{\mathrm{m} 0}$ and spectral wave period $T_{\mathrm{m}-1,0}$ on the basis of the three different model runs ( $\mathrm{P}$ for propagation with the phase-resolving wave model, $\mathrm{W}$ for wind wave growth with the phase-averaged wave model, and $\mathrm{N}$ for the wind wave model run with wind deactivated). 


$$
\begin{aligned}
H_{m 0, \mathrm{~T}} & =4 \sqrt{m_{0}^{P}+\left(m_{0}^{W}-m_{0}^{N}\right)} \\
& =\sqrt{H_{m 0, \mathrm{P}}^{2}+\left(H_{m 0, \mathrm{~W}}^{2}-H_{m 0, \mathrm{~N}}^{2}\right)} \\
T_{m-1,0, T}= & \frac{m_{-1}^{P}+\left(m_{-1}^{W}-m_{-1}^{N}\right)}{m_{0}^{P}+\left(m_{0}^{W}-m_{0}^{N}\right)} \\
= & \frac{H_{m 0, P}^{2} T_{m-1, \mathrm{P}}+\left(H_{m 0, W}^{2} T_{m-10, W}-H_{m 0, \mathrm{~N}}^{2} T_{m-10, \mathrm{~N}}\right)}{H_{m 0, \mathrm{P}}^{2}+\left(H_{m 0, \mathrm{~W}}^{2}-H_{m 0, \mathrm{~N}}^{2}\right)}
\end{aligned}
$$

\section{RESULTS OF THE HYBRID METHOD}

The hybrid method is illustrated for a typical offshore design wave condition with a significant wave height of 6 $\mathrm{m}$, a peak period of $12 \mathrm{~s}$ and a mean wave direction of $270^{\circ}$, a directional spreading of $25^{\circ}$, and a wind speed of 30 $\mathrm{m} / \mathrm{s}$. The applied models are the phase-resolving mild slope model PHAROS (Hurdle et al., 1984 and Deltares online documentation) and the phase-averaged wind wave model SWAN (Booij, et al., 1999). The extent of the computational grids of these models is illustrated in Figure 1. The western boundary of the PHAROS model is indicated with a black line. The boundary conditions for the PHAROS model are obtained from the SWAN model results along the black line. As can be seen, the extent of the SWAN model is much larger than that of the PHAROS model. This difference in size requires a careful handling of specifying the boundary conditions. As the above described method assumes equal sized computational areas, and the same wave boundary conditions for all three types of wave model runs, the boundary of the PHAROS model is taken as determining for comparing the results. This implies that the SWAN wave model run for the no wind condition $(\mathrm{N})$ should be performed with a modified wind in which the wind speed is set to zero for the area covered by the PHAROS model, whereas it remains constant outside this domain.

The PHAROS model is a mild slope model based on equations proposed by Berkhoff (1976). A basic PHAROS run computes the spatial variation of one wave component with a certain frequency and direction accounting for propagation, diffraction, reflection against quays, transmission over breakwaters, shoaling and dissipation by shallow water effects. As the wave boundary conditions for a typical harbor application are specified as a wave spectrum with frequency and direction spreading for a certain significant wave height, wave period and direction, the PHAROS model is applied for a set of wave components based on a discretization of the wave spectrum over frequency and direction. The contribution of each wave component to the penetrated wave field is obtained by weighting the results with the offshore wave variance in each single component. Summing all contribution then leads to the parameter $E_{\mathrm{P}}(\mathrm{x}, \mathrm{y})$.

The result of the combined PHAROS computation, for the above specified wave boundary condition is shown in Figure 2. This figure shows the spatial distribution of the significant wave height in the Port of IJmuiden. As can be seen the wave height decreases as the waves propagate further into the harbor basin. Near the entrance of the harbor the partial blocking effects of the breakwaters is clearly visible, as well as the refraction along the edges of the entrance channel. 


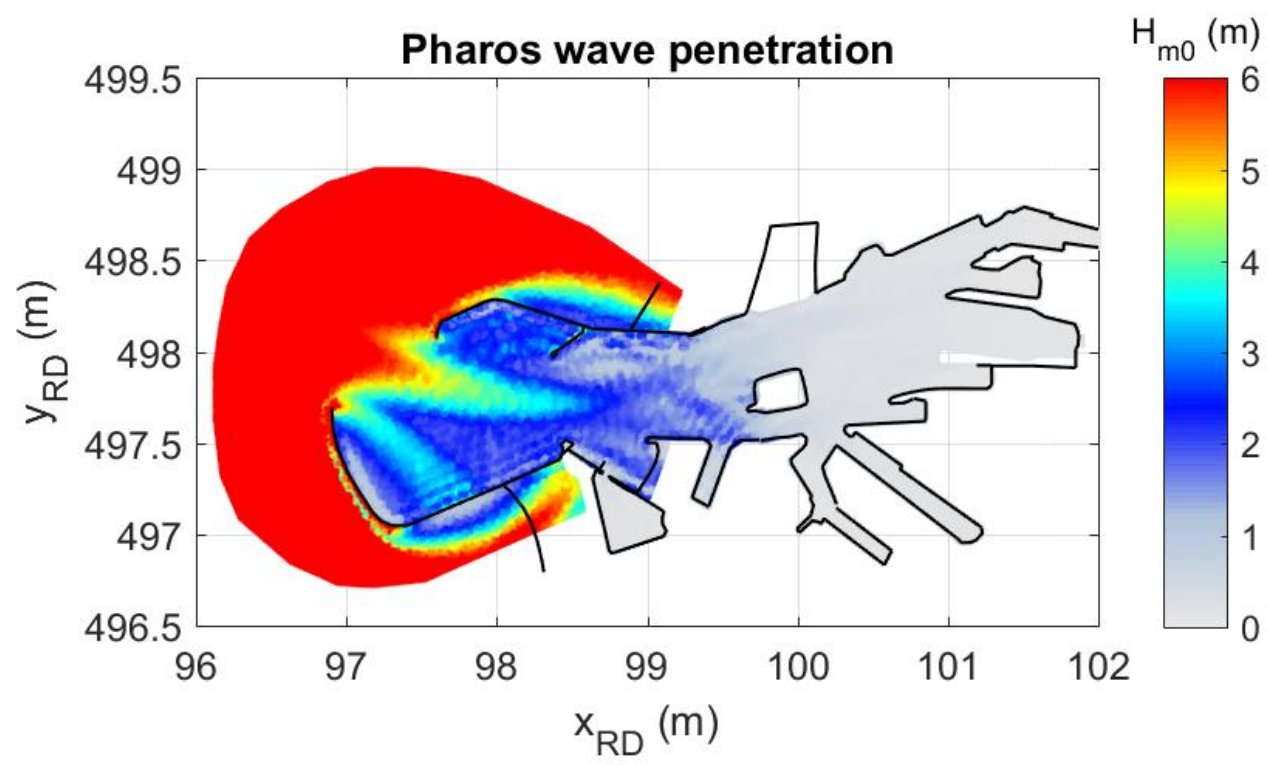

Figure 2 - Wave penetration into the Port of IJmuiden, computed with the PHAROS model.

The spatial distribution of the significant wave height based on a SWAN run with wind is visualized in Figure 3. The results in this figure show that the significant wave height in the harbor basin initially decreases after passing the breakwaters to increase again in eastward direction by the effect of wind. The arrows indicate the mean wave direction. It can be seen that refraction effects along the access channel turn the waves towards the sides of the channel. This effect acts as an effective filter for the longer wave components into the harbor basin.

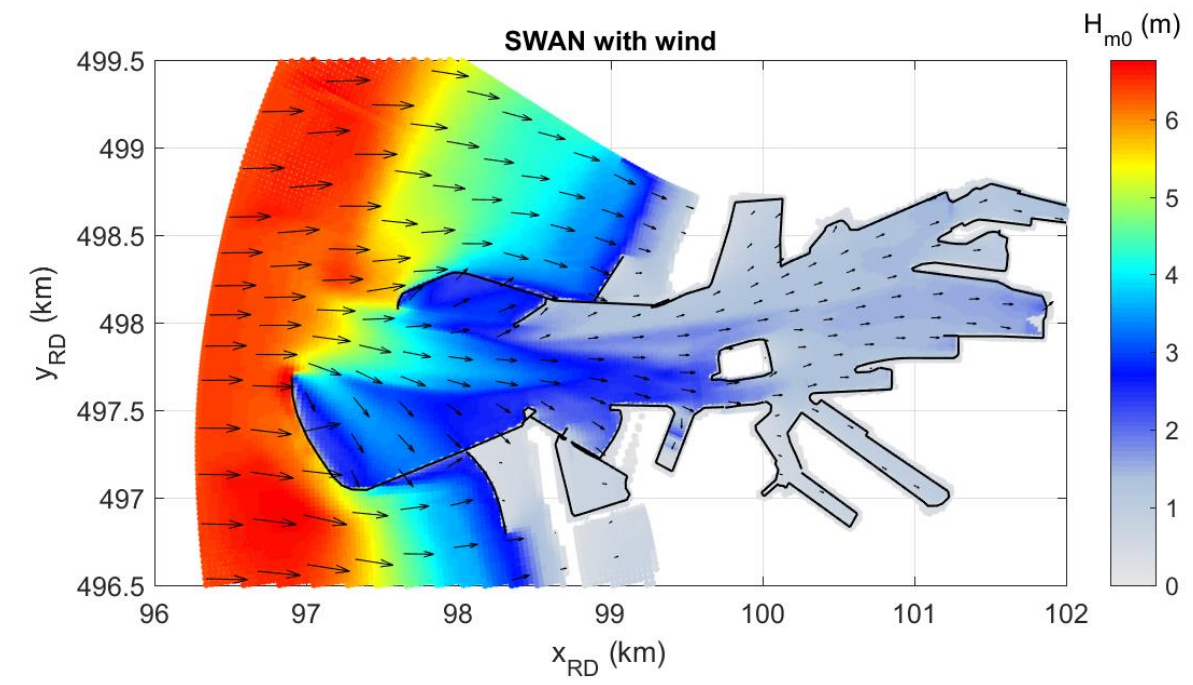

Figure 3 - Wave penetration and wind wave growth $\left(E_{w}\right)$ into the Port of IJmuiden computed with the SWAN model.

The spatial variation of the SWAN run of wave propagation without wind is shown in Figure 4 and the isolated effect of wave growth by wind is shown in Figure 5. As can be seen, the contribution of wind generation $\mathrm{G}$ increases in eastward direction. 


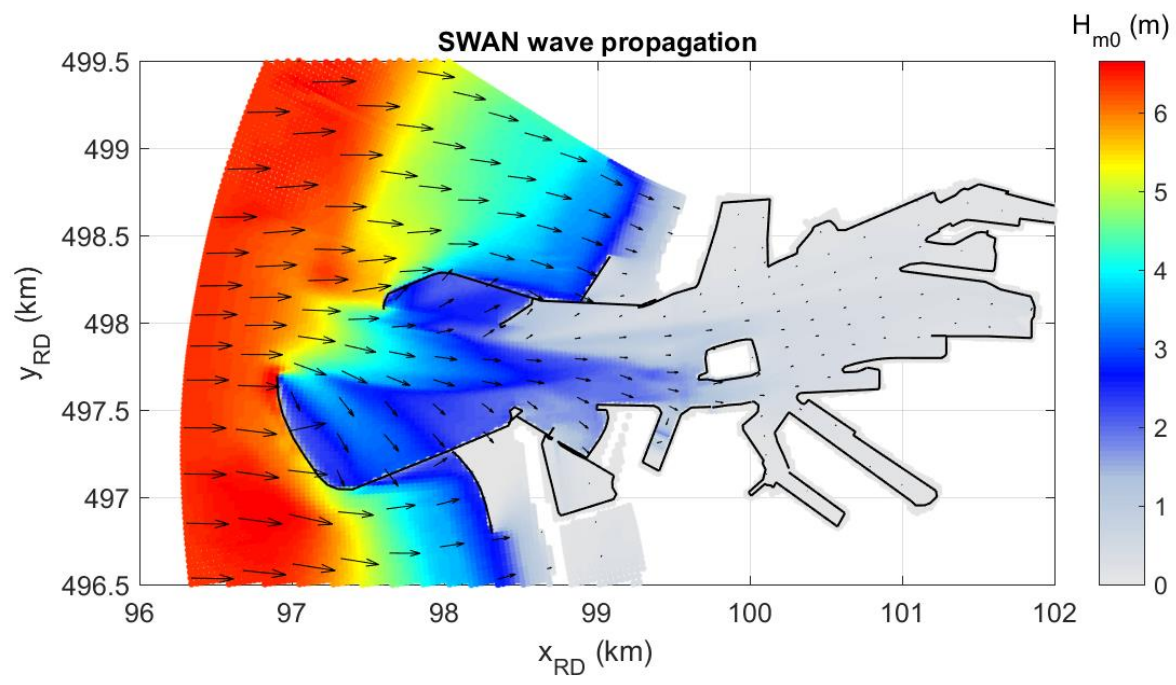

Figure 4 - Wave penetration with wind deactivated $\left(E_{N}\right)$ into the Port of IJmuiden computed with the SWAN model.

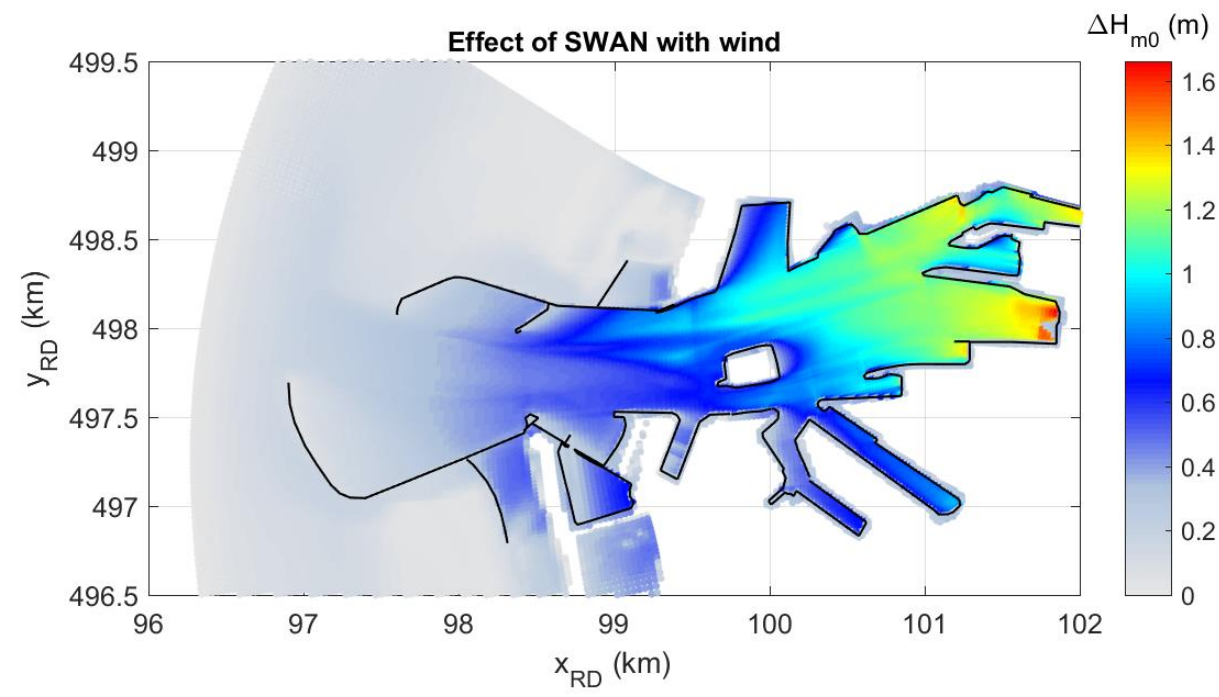

Figure 5 - Contribution of wind wave growth $\left(E_{G}\right)$ in the Port of IJmuiden, computed with the SWAN model. .

Comparison of these results with those shown in Figure 2, indicates that along the eastern boundary of the harbor basin the contribution of wind generation is significant and in this situation even dominant. In the present example, the wave penetration is computed with two different kinds of models. Of interest is the difference between the two models. This is shown in Figure 6, showing the difference between the PHAROS results $E_{P}$ and the SWAN results without wind $\mathrm{E}_{\mathrm{N}}$. Large differences occur in the shallow offshore area outside the breakwaters. This difference is due to the fact that the PHAROS model did not include dissipation effects, which were accounted for in the SWAN model. A striking difference occurs in the access channel between the breakwaters. Although refraction turns the waves to the sides of the access channel, more wave energy remains in the channel due to diffraction effect. Further into the harbor basin, the differences become smaller, also because the amount of penetrated wave energy is small in both the PHAROS and SWAN model results. 


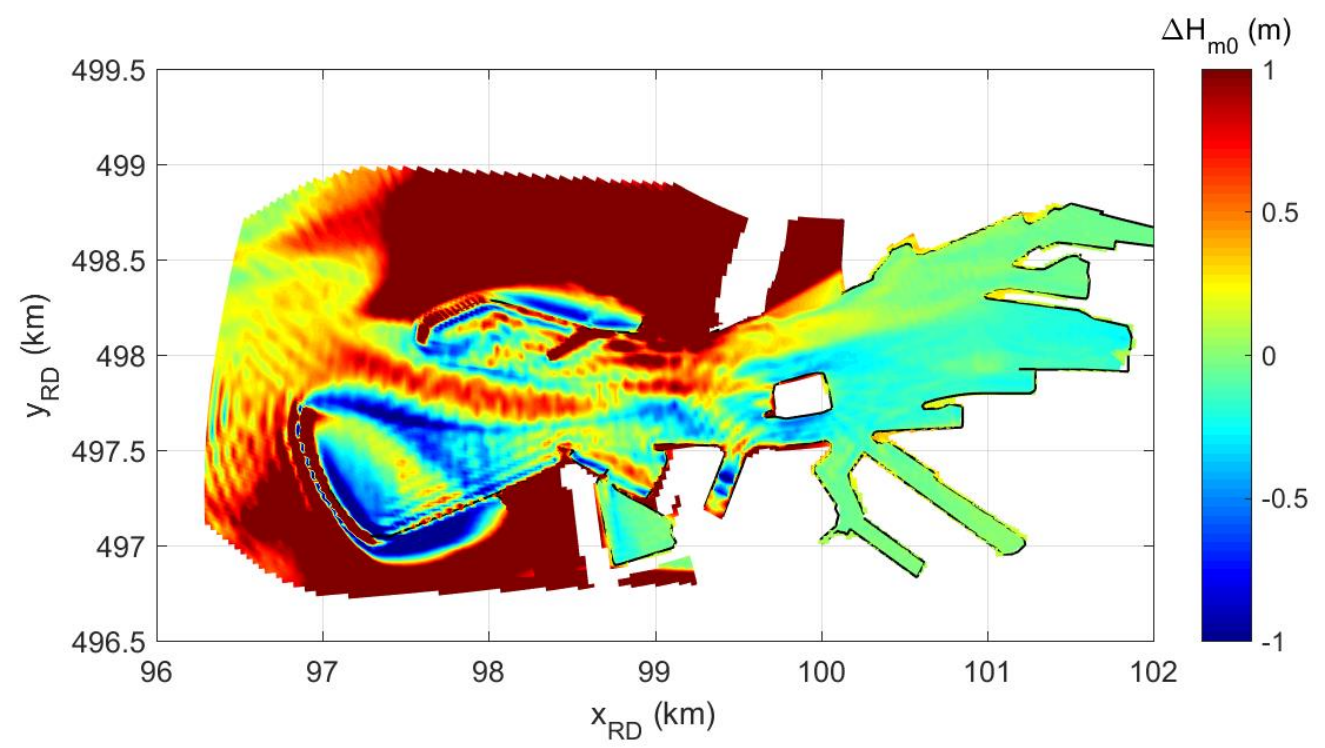

Figure 6 - Spatial variation of the difference between the wave penetration computed with the PHAROS model $\left(E_{P}\right)$ minus the results of the wave penetration computed with the SWAN model $\left(E_{N}\right)$.

As the waves penetrate into the harbor basin they remain under the influence of the wind. Close to the harbor entrance the effect of the wind is small, but this effect will increase in easterly direction. At the same time the penetrated wave height diminishes. This implies that depending on the position in the harbor area either the penetrated waves or the locally generated waves is dominant. Apart from the significant wave height, also the variation of the wave period is of interest. The variation of both wave parameters based on the results of the different kinds of wave model computation is shown in Figure 7 along a west to east transect in the harbor basin as indicated with the black dashed line in Figure 1. The range of $\mathrm{x}$-coordinates is chosen to match with the west-east extent of the computational domain of the PHAROS model.

The black line indicates the result of the SWAN model including wind (W). As can be seen the wave height and wave period diminish due to upwind fetch restrictions and refraction of wave energy to the sides of the access channel. For the last $2 \mathrm{~km}$, the waves start to grow again. The effect of wave penetration $(\mathrm{P})$ is indicated with the blue lines showing a steep drop of the significant wave height, whereas the wave period shows a slow decay and remains higher than the computation with wind activated. The contribution of local wind generation $(\mathrm{G})$ is shown with the red lines. For the significant wave height $\mathrm{H}_{\mathrm{m} 0}$, this line starts at zero and gradually increases to the same level as obtained with the SWAN model computation with wind. For the spectral period $\mathrm{T}_{\mathrm{m}-10}$, the line starts at about $6 \mathrm{~s}$, indicating that wind input acts on the existing wave field with a much higher wave period than when it would start from a zero condition. Hereafter, the wave period slowly diminishes to the same level as the one based on the SWAN model computation with wind.

The results for the last $2 \mathrm{~km}$ indicate that for this harbor area local wave growth is dominant and hardly affected by the penetrated wave. To validate this assumption, a SWAN wave model run was performed on the PHAROS domain in which a zero wave boundary condition was specified along the western boundary. The result of this computation shows the effect of local wave growth (indicated with the symbol L) and is illustrated in Figure 7 with the green lines. For both the significant wave height $\mathrm{H}_{\mathrm{m} 0}$ and the spectral wave period $\mathrm{T}_{\mathrm{m}-10}$, the results become practically equal to those computed with the SWAN model run with wind (W), confirming the assumption that in the eastern part of the harbour basin local wave growth is dominant. 

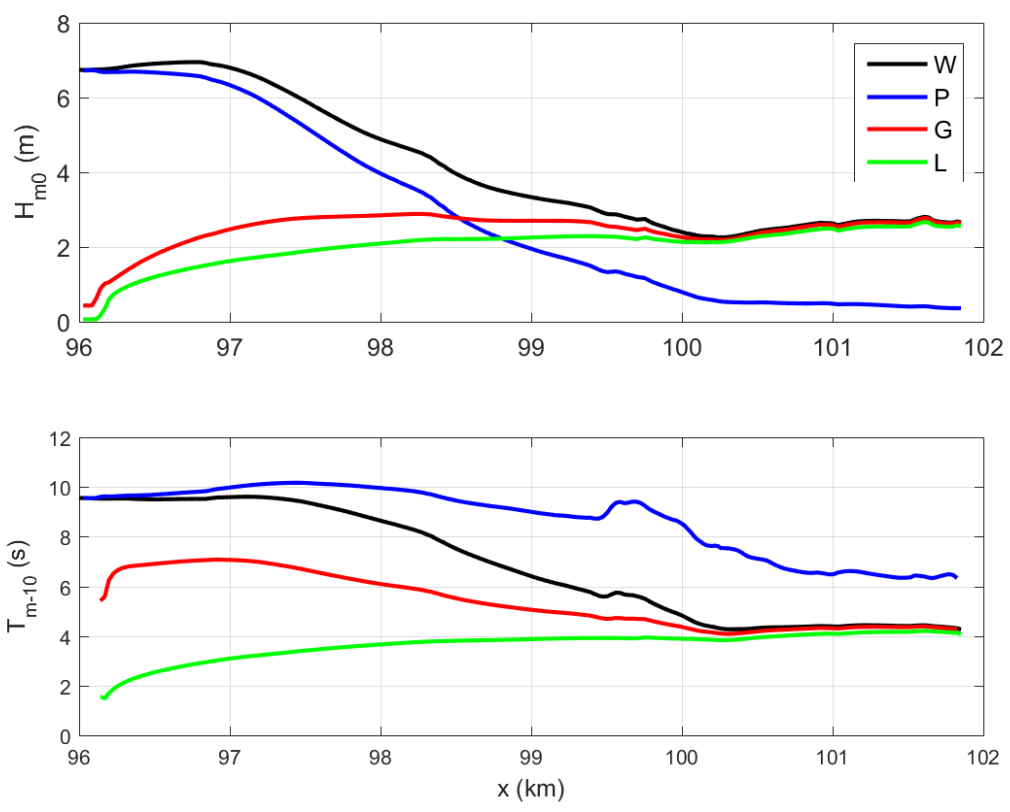

Figure 7 - Spatial variation of the significant wave height $\mathrm{H}_{\mathrm{m} 0}$ (upper panel) and spectral wave period $\mathrm{T}_{\mathrm{m}-10}$ (lower panel) for the cases of wind wave growth (black lines), wave penetration (blue lines), the isolated effect of wind wave growth (red lines) and local wave growth (green lines).

A closer look at the evolution of the wave field is by inspection of the frequency spectra at a number of locations along the west-east transect in the PHAROS domain (as indicated by the dashed black line in Figure 1). Figure 8 shows the frequency spectra at 6 locations for the conditions of wind wave growth (W), wave penetration $(\mathrm{P})$, the isolated effect of wind wave growth $(\mathrm{G})$ and local wave growth $(\mathrm{L})$. At the start of the domain (upper 2 panels), the spectra for the cases $\mathrm{W}$ and $\mathrm{P}$ are similar, indicating that the effect of wind is not yet noticeable. This also follows by the fact that the red and green lines are located on the $\mathrm{x}$-axis. At the next two locations $(\mathrm{x}=97.97 \mathrm{~km}$ and $x=99.16 \mathrm{~km}$ ), the effect of wind becomes noticeable and a new spectral peak develops at a period of about $6 \mathrm{~s}$. At $x=99.76 \mathrm{~km}$ a double peaked spectrum exist consisting of the penetrated and the locally generated waves. At the eastern end of the transect, a practically uni-modal spectrum remains, with a very small low frequency peak.
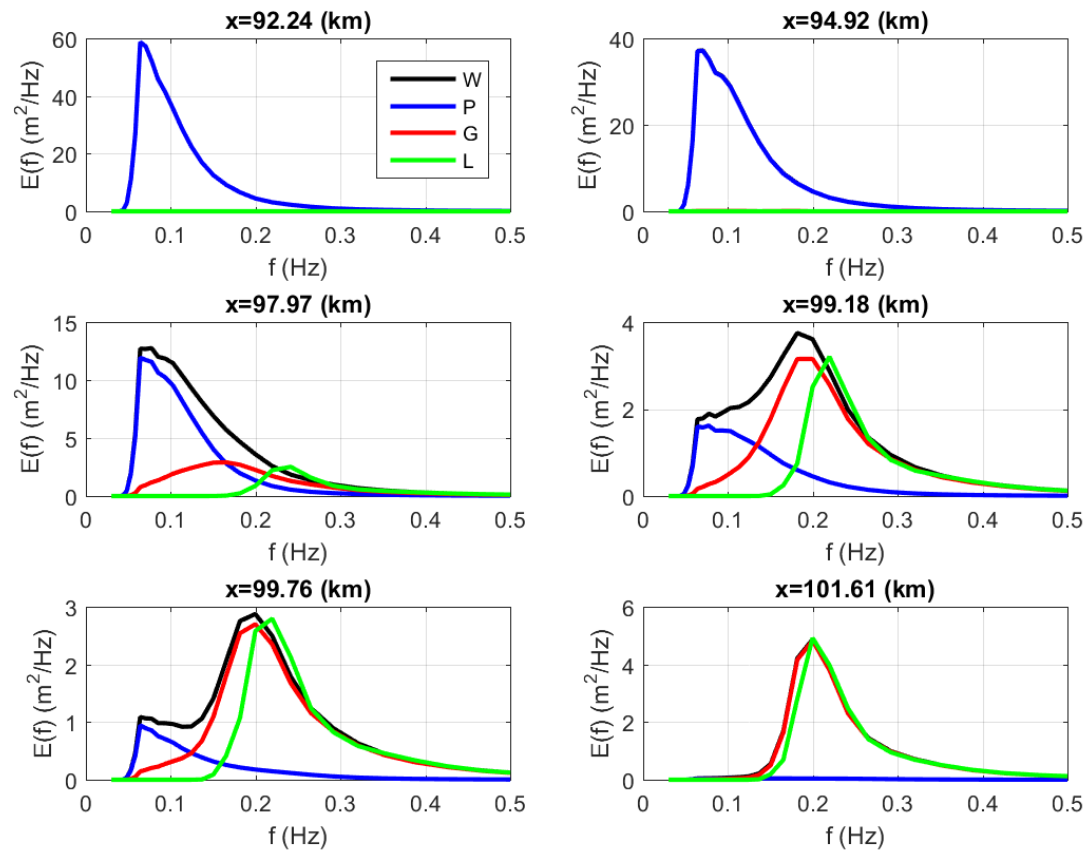

Figure 9 - Evolution of the frequency spectra along the west-east transect as a result of wind wave growth (black lines), wave penetration (blue lines), the isolated effect of wind wave growth (red lines) and local wave growth (green lines). 


\section{DISCUSSION}

The hybrid method is based on a number of assumptions. For instance, as the different model types are applied separately, effects of local generated waves on the diffraction of penetrated waves are neglected. On the other hand, effects of diffraction on wave generation are neglected. It is expected, however, that the errors due to these assumptions are insignificant.

Validation of this method is difficult due to scarcity of useful wave measurements in harbor basins. Especially as the most interesting measurement locations are located in the shipping lanes. Shore-based remote-sensing technique providing a spatial look may useful in validation studies. Adding the effect of local wave generation is especially relevant in large harbor basins. In small basins or in the area just behind the breakwaters, the effect of wave penetration is dominant over wind wave growth.

Modelling local wave growth in combination with penetrated waves gives additional challenges in choosing the right physical parameterizations for the effect of whitecapping dissipation in the phase-averaged wave model. In the spectra illustrated in Figure 9, the shape of the wave spectrum shows significant changes. Near the harbor entrance a uni-modal spectrum exists in equilibrium with the open sea wind conditions. Due to the wind a local wind sea develops with a much lower peak period resulting in a bi-modal wave spectrum. Finally, near the eastern edge only the local wind sea remains with a uni-modal spectrum. This situation reflects the problems associated with mixed sea states comprising of a background swell and a local wind sea. Parameterizations that scale the amount of whitecapping with a mean wave steepness are not suited as they tend to over-estimate the amount of local wave growth in the presence of a background low-frequency wave system, and conversely, the lower frequency wave dissipate too much.

\section{CONCLUSIONS}

A hybrid method was developed to determine wave conditions in large harbor basins accounting for the combined effect of wave penetration and local wind wave growth. The method uses a phase-resolving model to determine the effect of wave penetration and a phase-averaged wave model to determine the effect of local wind wave growth on the penetrating wave field. Both model types account for wave propagation, refraction, reflection, transmission and dissipation effects. The models differ in the sense that the phase-resolving model cannot account for local wave growth and that the phase-averaged model cannot account for diffraction effects. The key feature of the hybrid method is that the effect of local wave growth is determined by two model runs with the phase-averaged model. The first run includes wind effects while in the second run the wind is switched off. Subtracting the result enables isolating the effect of wave growth on top of the existing penetrating wave field. The results for the Port of IJmuiden show that local wind wave growth is the dominant process in the eastern part of the harbor basin. It is recommended to perform wave measurement in large harbor basins under strong wind conditions to validate the hybrid method.

\section{ACKNOWLEDGMENTS}

The author expresses his gratitude to fruitful discussions with David Hurdle and Olger Koop of Aktis Hydraulics, the Netherlands; Bernard Eikema and Harmen Talstra of Svašek Hydraulics, The Netherlands; and Robert Slomp of Rijkswaterstaat WVL, the Netherlands

\section{REFERENCES}

Booij, N., Ris, R.C., Holthuijsen, L.H., (1999). A third generation wave model for coastal regions. Part I, Model description and validation. J. Geophys. Res., 104, C4, 7649-7666.

Berkhoff, J.C.W. (1976). Mathematical models for simple harmonic linear water waves, wave diffraction and refraction, Delft Hydraulics Publication No. 163.

Chen, Q., Kaihatu, J.M., Hwang, P.A, (2004). Incorporation of Wind Effects into Bouusibeq Wave Models. Journal of Waterway, Port, Coastal, and Ocean Engineering, Vol. 130, No. 6, November 1, 2004. 312-321.

Deltares. https://www.deltares.nl/software/pharos.

Demirbiliek, Z., Zundel, A., Panchang, .V. (2010). Proc. Ports 2010: Builing on the Past, Respecting the Fiture. 121130.

Holthuijsen, L. H., Herman, A., Booij, N. (2003). Phase-decoupled refraction-diffraction for spectral wave models. Coastal Engineering, 49(4), 291-305. http://doi.org/https://doi.org/10.1016/S0378-3839(03)00065-6

Hurdle, D.P., Kostense, J.K., Van den Bosch, P. (1989). Mild-slope model for the wave behaviour in and around harbours and coastal structures in areas of variable depth and flow conditions. In: Advanced in water modeling and measurement.

SWAN team (2018), SWAN user manual. Delft University of Technology, the Netherlands. 